Maurício de Souza Arruda ${ }^{1}$

Hélo Sebastião Amâncio DE CAMARGO JÚNIOR ${ }^{2}$

Relato de caso

Palavras-chave

Gravidez ectópica/diagnóstico Gravidez ectópica/etiologia Gravidez ectópica/ultra-sonografia Cesárea/efeitos adversos

Miométrio

Ultra-sonografia pré-natal

Keywords

Pregnancy, ectopic/diagnosis

Pregnancy, ectopic/etiology Pregnancy, ectopic/ultrasonography Cesarean section/adverse effects

Myometrium

Ultrasonography, prenatal

\section{Gravidez ectópica na cicatriz uterina de cesárea: relato de caso}

\author{
Cesarean scar ectopic pregnancy: a case report
}

\section{Resumo}

Gravidez ectópica na cicatriz de cesárea é a forma mais rara de gravidez ectópica e provavelmente uma das mais perigosas em função dos riscos de ruptura e hemorragia volumosa. Essa situação deve ser diferenciada da gravidez cervical e de abortamento em curso, para que o tratamento apropriado seja imediatamente oferecido. Desde o advento da ultra-sonografia transvaginal, a gravidez ectópica na cicatriz de cesárea pode ser diagnosticada precocemente na gestação e, para isso o ultra-sonografista deve estar familiarizado com e atento aos critérios diagnósticos, especialmente em mulheres com cicatriz de cesárea prévia. Descrevemos aqui um caso de gravidez ectópica em cicatriz de cesárea, cujo diagnóstico foi tardio, havendo apresentação de involução espontânea.

\section{Abstract}

Ectopic pregnancy in a cesarean scar is the rarest form of ectopic pregnancy and probably the most dangerous one because of the risk of uterine rupture and massive hemorrhage. This condition must be distinguished from cervical pregnancy and spontaneous abortion in progress, so that the appropriate treatment can be immediately offered. Since the advent of endovaginal ultrasonography, ectopic pregnancy in a cesarean scar can be diagnosed early in pregnancy if the sonographer is familiarized with the diagnostic criteria of this situation, especially in women with previous cesarean scar. Here we describe a case of ectopic pregnancy in a cesarean scar in which the diagnosis was considerably late, with presentation of spontaneous regression.
Correspondência:

Maurício de Souza Arruda

Rua Louis Pasteur, 75, casa 16 - Parque Alto Taquard CEP 13087-773 - Campinas/SP E-mail:dr.arruda@uol.com.br

Recebido

$13 / 3 / 08$
Trabalho realizado no Hospital Estadual de Sumaré - HES, Sumaré (SP) e CDE Diagnóstico por Imagem - Campinas (SP), Brasil. ' Médico do Hospital Estadual de Sumaré, Ultra-sonografista do CDE Diagnóstico por Imagem - Campinas (SP), Brasil.

${ }^{2}$ Médico Radiologista do CDE Diagnóstico por Imagem - Campinas (SP), Brasil. 


\section{Introdução}

A implantação de uma gravidez na cicatriz de cesárea é considerada a apresentação mais rara de gravidez ectópica e de elevada morbimortalidade. A história natural desta condição é pouco conhecida, mas permitir a evolução da gestação carrega risco de ruptura da cicatriz uterina, possivelmente com necessidade de histerectomia ${ }^{1}$. Das várias teorias para explicar este tipo de gravidez ectópica, a mais razoável parece ser a de que o blastocisto penetra no miométrio através de uma microscópica deiscênci a na cicatriz, que pode ter sido criada por um trauma de uma incisão cesariana segmentar prévia ou qualquer outra cirurgia ${ }^{2}$.

A real incidência de gravidez em cicatriz de cesárea não é conhecida, em função dos poucos casos descritos na literatura: de 1978 a 2001 havia somente 18 casos publicados na literatura de língua inglesa e, entre 2002 e meados de 2003 , outros 25 casos foram publicados, dos quais 18 haviam ocorrido no mesmo centro $^{3,4}$. Em 2006, já havia 112 casos descritos na literatura, incluindo quatro séries de casos e vários relatos de casos isolados 5 .

Desde o advento da ultra-sonografia transvaginal, esse tipo de gravidez ectópica, que costumava ser tratada com histerectomia na maioria dos casos por extensa ruptura uterina, pôde ser diagnosticada precocemente na gestação e tratada de maneira conservadora, preservando-se a fertilidade ${ }^{6}$. Este artigo relata um raro caso de gravidez ectópica na cicatriz da cesárea, ilustrando os principais aspectos ultra-sonográficos.

\section{Relato de caso}

A paciente de 37 anos (G3 P2 A0), C2 foi encaminhada ao Hospital Estadual de Sumaré (HES) no dia 4 de outubro de 2007 com diagnóstico ecográfico de gestação anembrionada. A data da última menstruação era 2 de julho de 2007, tendo, portanto, tempo de amenorréia de 13 semanas e três dias. Apresentava um teste de $\beta$-HCG positivo datando de 7 de agosto de 2007. Já havia sido submetida a duas outras ultra-sonografias em clínica privada, com diagnóstico de gestação anembrionada. Os exames haviam sido realizados nos dias 20 de agosto e $1^{\circ}$ de setembro de 2007 , não tendo havido crescimento do saco gestacional neste período. A paciente fora orientada a aguardar a expulsão espontânea do saco gestacional. Em 3 de outubro de 2007, começou a apresentar sangramento vaginal em pequena quantidade e dor no hipogástrico, tipo cólica de fraca intensidade. No dia 4 de outubro, a terceira ultra-sonografia mostrou um saco gestacional irregular intracavitário medindo 1,7 x $1,5 \mathrm{~cm}$, sem evidência de embrião (Figura 1). Não foram encontradas alterações anexiais.
$\mathrm{Na}$ admissão ao hospital, a paciente encontrava-se em bom estado geral, corada, hidratada e afebril. Abdome discretamente doloroso no hipogástrio e descompressão brusca não dolorosa. No exame especular apresentava sangramento em pequena quantidade, vermelho vivo, sem restos. No toque bimanual, encontraram-se o útero levemente amolecido e doloroso e anexos com volume normal à palpação e indolores; colo impérvio. Foram realizados os exames de rotina: sorologia para sífilis (VDRL): negativo e tipagem sanguínea com fator Rh: O positivo.

A paciente foi submetida à curetagem uterina sob raquianestesia, sem intercorrências, com saída de pouca quantidade de material e muito sangramento. Histerometrias inicial e final de $11 \mathrm{~cm}$. A paciente recebeu alta hospitalar no dia seguinte.

No dia 19 de outubro, ela retornou ao HES referindo ter sangramento vaginal intermitente, ora em maior quantidade, ora em menor quantidade, desde a curetagem uterina, e já trazia uma nova ultra-sonografia realizada no mesmo dia e que mostrava "imagem complexa com áreas econegativas medindo $3,2 \times 3,0 \times 2,5 \mathrm{~cm}$, sugestivas de restos placentários, e um mioma uterino na parede anterior com maior diâmetro medindo $3,5 \mathrm{~cm}$ ”. O próprio serviço de ultra-sonografia encaminhou a paciente novamente para o HES com o diagnóstico de abortamento incompleto.

Nessa segunda admissão, a paciente apresentava-se em bom estado geral, levemente descorada, hidratada e afebril. Apresentava abdome plano, normotenso e discretamente doloroso no hipogástrio e descompressão brusca não dolorosa, além de especular com pequena quantidade de sangramento vermelho vivo, sem sinais de restos placentários. Ao toque bimanual, achou-se útero de tamanho normal, discretamente

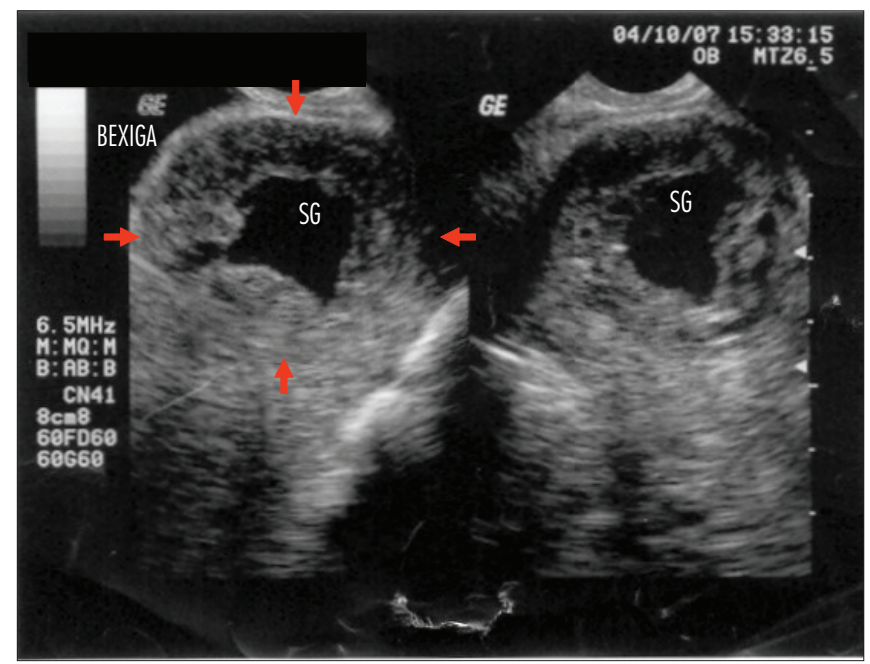

Figura 1 - Corte longitudinal do útero mostrando imagem que foi interpretada como saco gestacional (SG, delimitado pelas setas vermelhas) irregular, tópico, sem embrião ou vesícula vitelina em seu interior. Saco gestacional irregular. Ausência de tecido miometrial normal na porção anterior, onde se vê uma pequena porção da bexiga, assim como é nítida uma porção de miométrio posterior à reação tecidual 
doloroso à palpação, bem como anexos palpáveis e de volume normal, indolores. Não apresentava abaulamento ou dor no fundo de saco posterior. Foi colhida uma amostra de sangue para a realização de um hemograma, que mostrou: $3,2 \times 10^{6}$ glóbulos vermelhos $/ \mathrm{mL}$, hematócrito de 30,2\% e hemoglobina de 10,2 g/dL. Leucócitos: $8.300 \mathrm{~mm}^{3}$, sem desvio à esquerda.

Como a curetagem uterina havia transcorrido sem intercorrências e sem dificuldades técnicas, com saída de pequena quantidade de material e muitos coágulos, e como o colo sempre se manteve impérvio, suspeitamos tratar-se de gestação ectópica. Essa suspeita foi confirmada com o resultado anatomopatológico do material da curetagem que mostrou endométrio decidualizado, sem vilosidades coriônicas e com o resultado positivo para $\beta$-HCG plasmático, dosado nessa internação, com valor de $51 \mathrm{mUI} / \mathrm{mL}$.

Como a paciente estava hemodinamicamente estável e com melhora significativa do sangramento vaginal, uma nova ultra-sonografia foi agendada para o dia seguinte, sendo realizada em 20 de outubro de 2007 . O exame mostrou útero com volume estimado de $160 \mathrm{~mL}$, cavidade endometrial vazia, endométrio com espessura de $5 \mathrm{~mm}$; na topografia de segmento uterino, na parede anterior, presença de uma imagem hipoecogênica medindo $4,3 \times 2,9 \mathrm{~cm}$, heterogênea, que abaulava nitidamente a serosa sem ter contato com o endométrio, com uma área cística em seu interior medindo 1,7 x 1,3 cm (interpretada como saco gestacional). Observou-se ausência de fluxo ao mapeamento com doplerfluxometria colorida, ausência de tecido miometrial normal entre a bexiga e o saco gestacional (Figuras 2 e 3). Ambos os ovários apresentavam aspecto ecográfico normal, sendo que a cavidade abdominal estava ausente de líquido livre. Os achados ecográficos foram interpretados como gestação

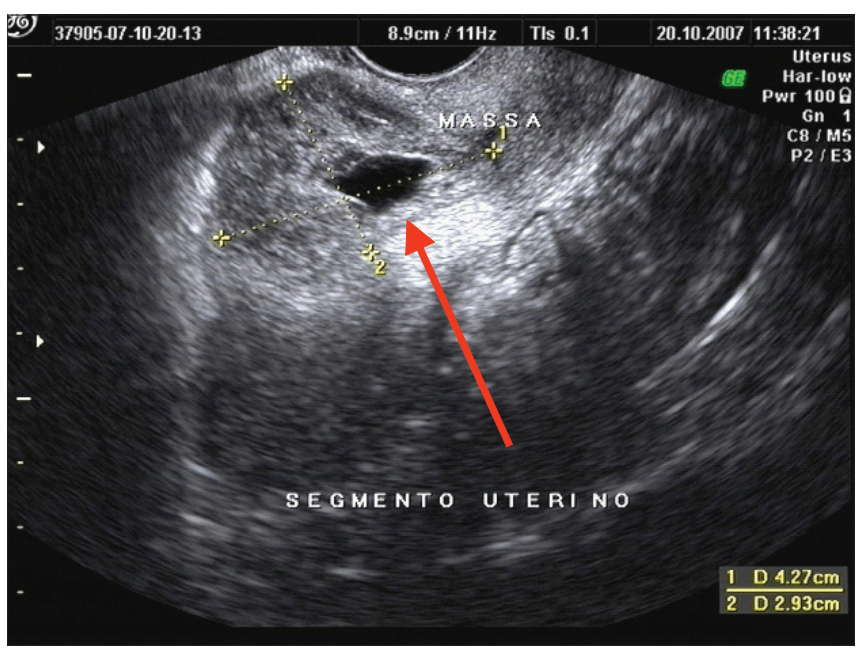

Figura 2 - Corte longitudinal do útero mostrando massa heterogênea na parede anterior do segmento uterino e, em seu interior, saco gestacional já bastante irregular. ectópica na cicatriz de cesárea uma vez que preenchiam todos os critérios para esta condição.

A paciente foi informada das opções terapêuticas e sempre rejeitou enfaticamente o tratamento cirúrgico. Assim, instituímos conduta expectante, considerando a possibilidade de involução espontânea da gestação. A paciente também foi informada dos riscos desta opção terapêutica e da importância do rigoroso monitoramento clínico e ecográfico, inicialmente semanal, até que estivéssemos convencidos de que a massa trofoblástica estivesse involuindo; a partir de então, os controles seriam a cada três ou quatro semanas. Foi orientada, a retornar imediatamente ao HES se sentisse dor abdominal ou tivesse sangramento vaginal e, ainda, sobre eventual necessidade de histerectomia de urgência.

O primeiro retorno para reavaliação ecográfica foi feito em 26 de outubro de 2007, uma semana após o exame anterior, quando a paciente se encontrava assintomática e sem sangramento. As dimensões da gravidez ectópica estavam discretamente diminuídas em relação ao exame anterior: 3,7 x 2,4 cm, e o aspecto era mais heterogêneo com múltiplas áreas císticas em seu interior (Figura 4). Não havia líquido livre na cavidade. Nessa ocasião orientamos o uso de desogestrel contínuo com método contraceptivo, pois a paciente referia intolerância às pílulas anticoncepcionais orais combinadas. Foi dosado $\beta$-HCG plasmático quantitativo em 23 de outubro de 2007: $17 \mathrm{mUI} / \mathrm{mL}$.

Em 9 de novembro de 2007, a paciente mantinha-se assintomática e sem sangramento. A gravidez ectópica media $3,1 \times 2,8 \mathrm{~cm}$ e, portanto, discretamente menor em relação aos exames anteriores (Figura 5). Em 23 de novembro de 2007, a massa trofoblástica media $3,2 \times 2,6 \mathrm{~cm}$. Novo $\beta$-HCG quantitativo em 24 de novembro de 2007: <1,0 mUI/mL. Em 25 de janeiro de 2008, a paciente voltou a aprsentar sangramento vaginal;

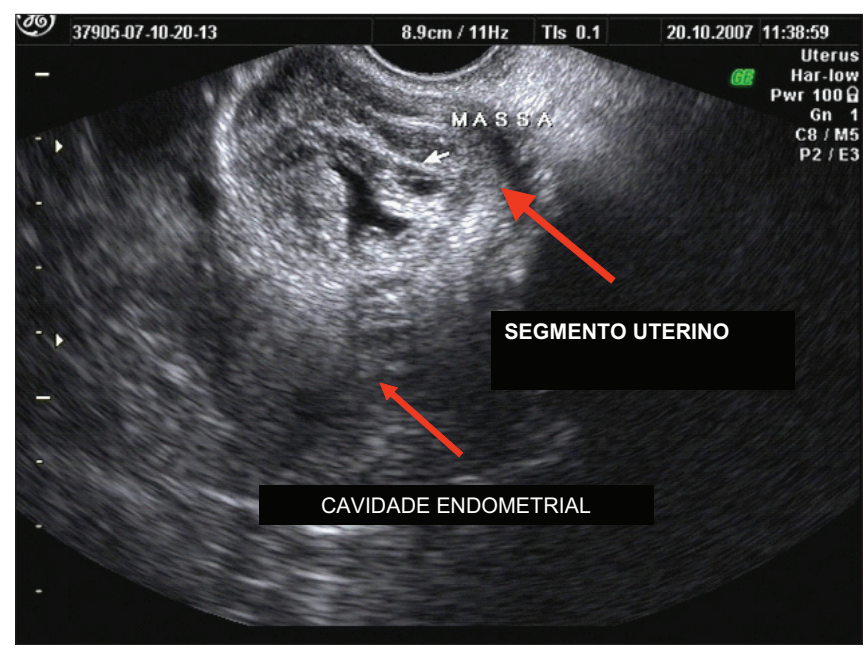

Figura 3 - Corte transversal do útero mostrando massa heterogênea na parede anterior do segmento uterino, sem contato com a cavidade endometrial. 


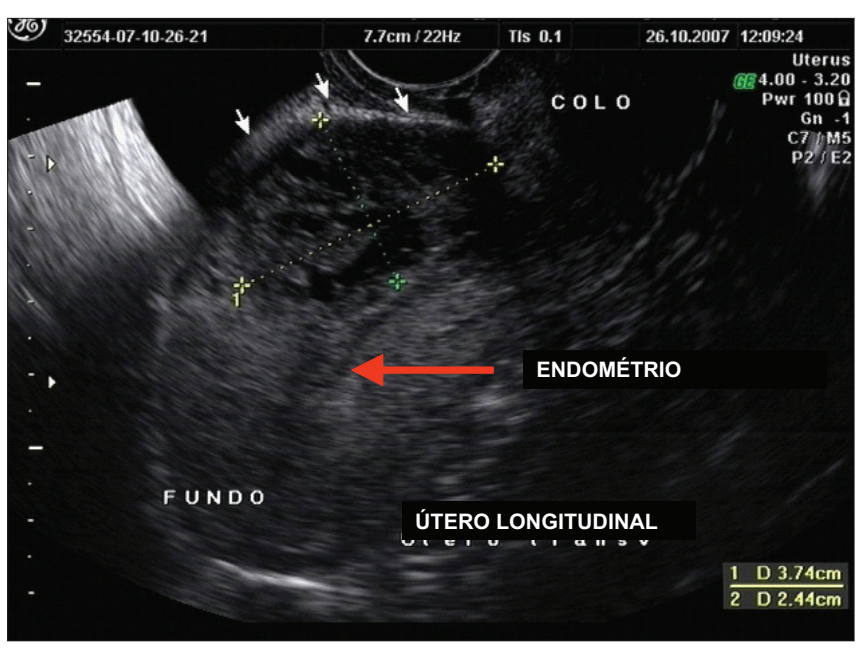

Figura 4 - Corte longitudinal do útero seis dias após o exame diagnóstico, mostrando discreta redução das dimensões da gravidez ectópica. Já não se identifica o saco gestacional. Cavidade endometrial vazia.

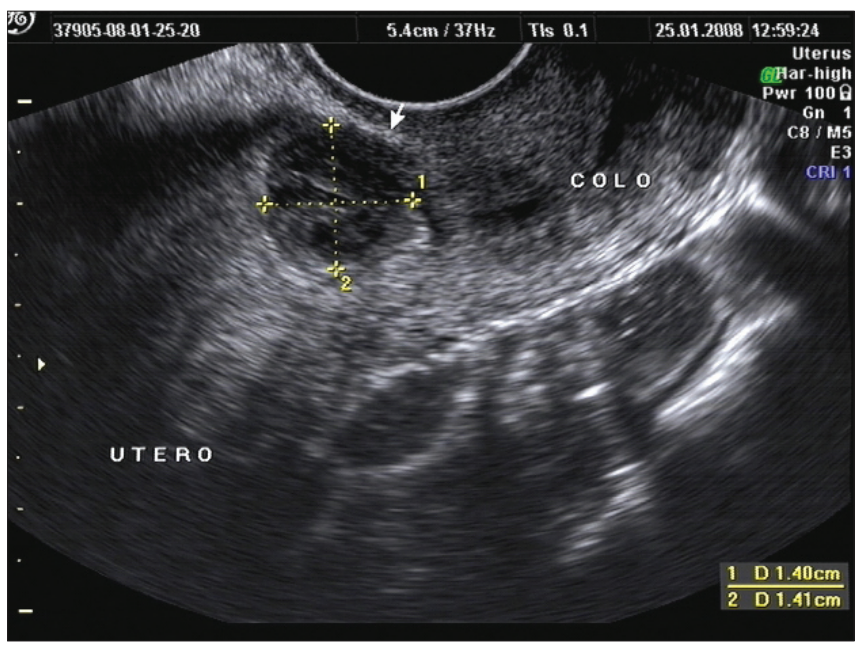

Figura 6 - Corte longitudinal do útero três meses após o exame diagnóstico. Com a involução da massa trofoblástica, ficou mais evidente a localização no segmento desta gestação ectópica.

havia suspendido o uso de desogestrel por estar sentindo cefaléia com a medicação. Repetimos o exame ultra-sonográfico nesse dia e a massa trofoblástica media 1,4 x 1,4 $\mathrm{cm}$ (Figura 6). O endométrio estava regular, homogêneo com espessura de $5 \mathrm{~mm}$. Reforçamos a importância do uso de métodos contraceptivos e orientamos preservativo, uma vez que a paciente também rejeitava a opção de contraceptivo injetável. Em 29 de fevereiro de 2008, a massa trofoblástica já estava praticamente imperceptível, apresentando-se como pequeno nódulo hipoecogênico no segmento uterino medindo 0,9 x 0,6 cm (Figura 7). A paciente afirmou estar menstruando regularmente, com cólicas moderadas. Nessa ocasião, questionou sobre os riscos de uma nova gestação, pois gostaria de ter mais um filho. Expusemos não só o risco, ainda que fosse remoto, de uma nova gravidez ectópica, mas principalmente de uma rotura espontânea do segmento uterino em uma

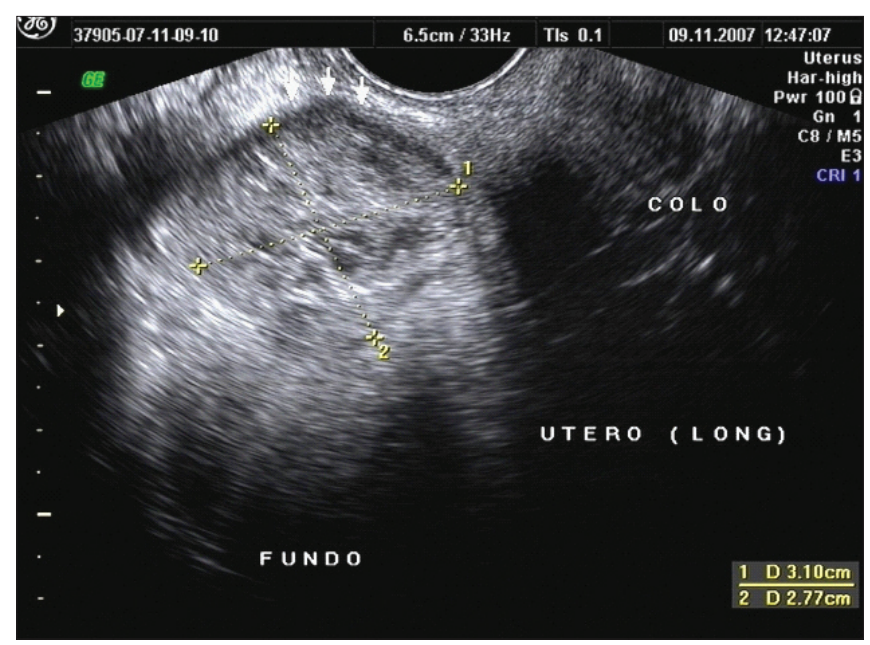

Figura 5 - Corte longitudinal do útero 20 dias após o exame diagnóstico, com tecido trofoblástico em involução.

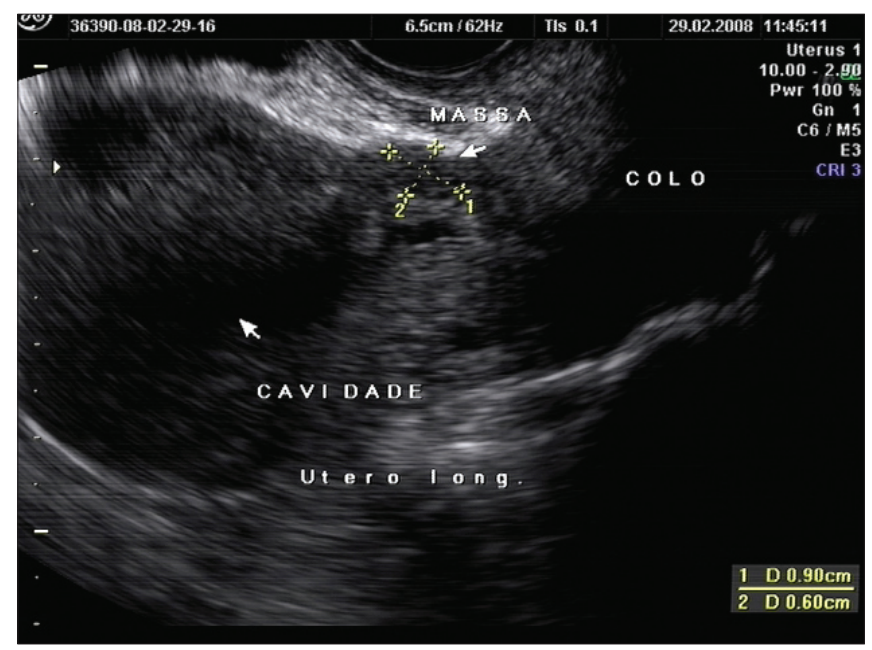

Figura 7 - Corte longitudinal do útero quatro meses após o exame diagnóstico. Massa trofoblástica quase imperceptível no segmento uterino.

gestação tópica, mesmo antes de entrar em trabalho de parto. Nossa recomendação foi procurar o serviço de planejamento familiar para esterilização cirúrgica, quer fosse ligadura tubária, ou vasectomia para o marido. A paciente foi dispensada de novos controles ecográficos e encaminhada para uma Unidade Básica de Saúde (UBS) para exames de rotina. Recomendamos, também, que se houvesse atraso menstrual ou suspeita de gravidez, ela deveria procurar imediatamente o HES.

\section{Discussão}

Embora o uso da ultra-sonografia transvaginal no diagnóstico da gravidez ectópica esteja bem estabelecido, o diagnóstico diferencial entre abortamento espontâneo em curso, gravidez cervical e gravidez na cicatriz da cesárea não é tão fácil ${ }^{7}$. Rígidos critérios ultra-sonográficos 
devem ser preenchidos para se estabelecer tal diagnóstico: cavidade uterina vazia, canal cervical vazio, desenvolvimento do saco gestacional da parede anterior da parede do istmo e ausência de tecido miometrial normal entre a bexiga e o saco gestacional. Esse último critério permite o diagnóstico diferencial de uma gravidez cervical ${ }^{6}$.

Quanto ao caso apresentado, o diagnóstico ecográfico inicial foi de gestação tópica anembrionada.

Posteriormente, coágulos na cavidade uterina foram interpretados como restos ovulares e a gestação ectópica na cicatriz da cesárea, nesta ocasião já com debris em seu interior, próprio do processo de involução, foi interpretada como um mioma subseroso.

Somente na segunda internação, quando a paciente retornou para o HES para nova curetagem por abortamento incompleto, havia elementos para forte suspeita de gestação ectópica, especialmente a ausência de restos placentários no material de curetagem e ausência de vilosidades coriônicas no estudo anatomopatológico do conteúdo da cavidade endometrial, além da persistência do sangramento vaginal e $\beta$-HCG plasmático ainda positivo.

Nesse caso, o diagnóstico de gravidez ectópica na cicatriz da cesárea foi feito muito tardiamente (tempo de amenorréia de 15 semanas e cinco dias). O nível de $\beta$-HCG já estava muito baixo e não havia fluxo trofoblástico ao mapeamento com Doppler colorido, indicando que a gestação ectópica já estava regredindo espontaneamente. Como a paciente rejeitou a proposta cirúrgica, foi aplicado o conhecimento já amplamente divulgado para tratamento expectante de gravidez ectópica ${ }^{8}$. Como a paciente preenchia todos os critérios para conduta expectante, prescindindo inclusive da aplicação de metotrexate, foi adotada a conduta de acompanhamento clínico com rigoroso controle ecográfico.

Por causa da raridade desse tipo de gravidez ectópica e das freqüentes, e graves, complicações quando não tratada adequadamente, a recomendação atual mais freqüente diante desse diagnóstico é a interrupção mais precoce da gestação, visando eliminar o conteúdo gestacional e, sempre que desejável, preservar a fertilidade, embora não haja um protocolo bem estabelecido para condução desses casos ${ }^{9}$.

A mais recente revisão de literatura sobre o tema mostra que a administração sistêmica de metotrexate é a alternativa apropriada para as pacientes com nível sérico de $\beta$-HCG inferior a $5.000 \mathrm{mUI} / \mathrm{mL}$, com gravidez ectópica íntegra e hemodinamicamente estável. A administração local de metotrexate, cloreto de potássio e solução hiperosmolar, via abdominal ou transvaginal e guiada por ultra-sonografia, também foi descrita com resultados satisfatórios. Com o tratamento medicamentoso, mesmo após a interrupção da gestação, podem persistir dor abdominal e sangramento vaginal, às vezes abundante. Nesses casos, a aspiração cirúrgica do conteúdo trofoblástico guiado por ultra-sonografia, evita, muitas vezes, a laparotomia e a histerectomia ${ }^{10}$.

A curetagem uterina não deve ser indicada como tratamento nessa situação em função da elevada taxa de insucesso na remoção do tecido trofoblástico e da alta freqüência de complicações graves, com o risco de rotura uterina com hemorragia grave e histerectomia de urgência, além da possibilidade de perfuração vesical ${ }^{5,10}$.

Laparotomia com ressecção de todo o tecido trofoblástico deve ser sempre considerada para as pacientes que apresentarem falhas no tratamento medicamentoso e das quais o diagnóstico foi muito tardio. O emprego da histerectomia foi relatada em pelo menos 13 casos até novembro de 2006 tanto como primeira opção terapêutica como por insucesso de outras modalidades terapeûticas ${ }^{11}$.

Wang et al. ${ }^{12}$ propuseram, para pacientes com gravidez ectópica íntegra, após oito casos bem sucedidos, sem necessidade de conversão para laparotomia, a ressecção de todo o tecido trofoblástico através da vídeolaparoscopia e sutura endoscópica com pontos separados de ácido poliglicólico 2-0 das bordas sadias do miométrio.

A conduta expectante adotada em muitos casos, pela expectativa de que pudesse evoluir até o termo ou por ter sido inicialmente interpretada como abortamento em curso, revelou elevadas taxas de complicações graves 5 . E, de forma semelhante ao que foi aqui apresentado, há casos descritos na literatura nos quais o diagnóstico foi feito já na fase de abortamento ( $\beta$-HCG declinando, saco gestacional sem embrião ou embrião sem atividade cardíaca) e que também foram acompanhados sem intervenção medicamentosa ou cirúrgica, com involução da massa trofoblástica e sem complicações ${ }^{4,9}$. O cuidado de orientar enfaticamente a paciente quanto à necessidade de controle ecográfico semanal e a recomendação de retornar ao hospital imediatamente caso apresentasse dor ou sangramento são plenamente justificáveis em função dos riscos mencionados.

Quanto à possibilidade de nova gestação, os riscos associados a uma eventual nova gravidez estariam associados mais à idade materna (no caso aqui descrito) do que ao antecedente de gravidez ectópica na cicatriz uterina das cesáreas anteriores, embora houvesse a possibilidade de recorrência. Ben Nagi et al. ${ }^{13}$ apresentaram informações sobre 24 pacientes que desejaram engravidar após terem tido este tipo de gravidez ectópica: 21 (88\%) engravidaram novamente; 20 gestações foram intra-uterinas e uma paciente apresentou nova gravidez ectópica na cicatriz uterina da cesárea. Dentre as gestações intra-uterinas, sete $(35 \%)$ terminaram em abortamento espontâneo ainda no primeiro trimestre. Em nove casos indicou-se parto cesárea eletivo, sem complicações, após a $37^{a}$ semana de gestação. Duas gestações ainda estavam em curso e não havia informações sobre as duas outras gestações até a 
publicação do artigo. Três pacientes não engravidaram novamente.

O aumento do número de casos de gravidez ectópica na cicatriz de cesárea pode estar relacionado ao significativo aumento de partos abdominais em todo o mundo e também à melhora da acurácia diagnóstica com a ultra-sonografia transvaginal. O ultra-sonografista deve sempre considerar essa possibilidade em toda avaliação obstétrica do primeiro trimestre, especialmente em mulheres com cesarianas anteriores. É preciso, também, conhecer os critérios diagnósticos dessa situação e para que seja possível diferenciá-la de um abortamento em curso ou de uma gravidez cervical e para que, dessa forma, a conduta apropriada seja tomada em cada caso, evitando-se complicações graves.

\section{Referências}

1. Fylstra DL, Pound-Chang T, Miller MG, Cooper A, Miller KM. Ectopic pregnancy within a cesarean delivery scar: a case report. Am J Obstet Gynecol. 2002;187(2):302-4.

2. Cheng PJ, Chueh HY, Soong YK. Sonographic diagnosis of a uterine defect in a pregnancy at 6 weeks' gestation with a history of curettage. Ultrasound Obstet Gynecol. 2003;21 (5):501-3.

3. Fylstra DL. Ectopic pregnancy within a cesarean scar: a review. Obstet Gynecol Surv. 2002;57(8):537-43.

4. Jurkovic D, Hillaby K, Woelfer B, Lawrence A, Salim R, Elson CJ. First-trimester diagnosis and management of pregnancies implanted into the lower uterine segment cesarean section scar. Ultrasound Obstet Gynecol. 2003;21(3):220-7.

5. Rotas MA, Haberman S, Levgur M. Cesarean scar ectopic pregnancy: etiology, diagnosis, and management. Obstet Gynecol. 2006;107(6):1373-81

6. Godin PA, Bassil S, Donnez J. An ectopic pregnancy developing in a previous caesarian section scar. Fertil Steril. 1997;67(2): $398-400$

7. Noguchi S, Adachi M, Konishi M, Habara T, Nakatsuka M, Kudo T. Intramural pregnancy in a previous cesarean section scar: a case report on conservative surgery. Acta Obstet Gynecol Scand. $2005 ; 84(5): 493-5$.

8. Han KK, Elito Júnior J, Camano L. Conduta expectante para gravidez tubária íntegra. Rev Bras Ginecol Obstet. 1999;21 (8):465-70.

9. Elito Júnior J, Montenegro NAMM, Soares RC, Camano L. Gravidez ectópica não rota: diagnóstico e tratamento. Situação atual. Rev Bras Ginecol. Obstet. 2008;30(3): 149-59.

10. Maymon R, Halperin R, Mendlovic S, Schneider D, Herman A. Ectopic pregnancies in a caesarean scar: review of the medical approach to an iatrogenic complication. Hum Reprod Update. $2004 ; 10(6): 515-23$

11. Ash A, Smith A, Maxwell D. Caesarean scar pregnancy. BJOG. 2007; $114(3): 253-63$

12. Wang YL, Su TH, Chen HS. Operative laparoscopy for unruptured ectopic pregnancy in a caesarean scar. BJOG. 2006; $113(9): 1035-8$

13. Ben Nagi J, Helmy S, Ofili-Yebovi D, Yazbek J, Sawyer E, Jurkovic D. Reproductive outcomes of women with a previous history of caesarean scar ectopic pregnancies. Hum Reprod. 2007;22(7):2012-5. 